\title{
Bounds on the Distinguishing Chromatic Number
}

\author{
Karen L. Collins \\ Department of Mathematics and Computer Science \\ Wesleyan University, Middletown, CT 06459-0128 \\ kcollins@wesleyan.edu \\ Mark Hovey \\ Department of Mathematics and Computer Science \\ Wesleyan University, Middletown, CT 06459-0128 \\ mhovey@wesleyan.edu \\ Ann N. Trenk* \\ Department of Mathematics \\ Wellesley College, Wellesley, MA 02481 \\ atrenk@wellesley.edu
}

Submitted: Apr 11, 2008; Accepted: Jul 8, 2009; Published: Jul 24, 2009

Mathematics Subject Classifications: 05C15, 05C25

\begin{abstract}
Collins and Trenk define the distinguishing chromatic number $\chi_{D}(G)$ of a graph $G$ to be the minimum number of colors needed to properly color the vertices of $G$ so that the only automorphism of $G$ that preserves colors is the identity. They prove results about $\chi_{D}(G)$ based on the underlying graph $G$. In this paper we prove results that relate $\chi_{D}(G)$ to the automorphism group of $G$. We prove two upper bounds for $\chi_{D}(G)$ in terms of the chromatic number $\chi(G)$ and show that each result is tight: (1) if $\operatorname{Aut}(G)$ is any finite group of order $p_{1}^{i_{1}} p_{2}^{i_{2}} \cdots p_{k}^{i_{k}}$ then $\chi_{D}(G) \leq \chi(G)+i_{1}+i_{2} \cdots+i_{k}$, and (2) if $\operatorname{Aut}(G)$ is a finite and abelian group written $\operatorname{Aut}(G)=\mathbf{Z}_{p_{1}^{i_{1}}} \times \cdots \times \mathbf{Z}_{p_{k}^{i_{k}}}$ then we get the improved bound $\chi_{D}(G) \leq \chi(G)+k$. In addition, we characterize automorphism groups of graphs with $\chi_{D}(G)=2$ and discuss similar results for graphs with $\chi_{D}(G)=3$.
\end{abstract}

\section{Introduction}

The distinguishing number $D(G)$ of a graph was first defined by Albertson and Collins [1] as the minimum number of colors needed to color the vertices of $G$ so that the only

${ }^{*}$ The third author's work was supported in part by a Wellesley College Brachman Hoffman Fellowship. 
automorphism of $G$ that preserves colors is the identity. The distinguishing number of the cycle $C_{n}$ is the answer to the following question which inspired the definition of $D(G)$ : given a ring of seemingly identical keys that open different doors, how many colors are needed to distinguish them? The subject has received considerable attention since then (e.g., see $[1,2,9,14])$.

In the definition of the distinguishing number, there is no requirement that the coloring be proper. Indeed, in labeling keys on a key ring, there is no reason why adjacent keys must receive different colors. However, in other graph theory questions where edges in a graph represent conflicts (scheduling meetings, storing chemicals, etc.) a proper coloring is needed, and one with a small number of colors is desirable. If this coloring is also distinguishing we can identify the objects represented by the vertices just by looking at the graph and its coloring.

Collins and Trenk [4] define the distinguishing chromatic number which incorporates the additional requirement that the labeling be proper.

Definition 1.1 A labeling of the vertices of a graph $G, h: V(G) \rightarrow\{1, \ldots, r\}$, is said to be proper $r$-distinguishing (or just proper distinguishing) if it is a proper labeling (i.e., coloring) of the graph and no automorphism of the graph preserves all of the vertex labels. The distinguishing chromatic number of a graph $G$, denoted by $\chi_{D}(G)$, is the minimum $r$ such that $G$ has a proper $r$-distinguishing labeling.

For example, the graph $H$ in Figure 2 at the end of Section 3 has $D(H)=2$ (labeling vertex $w_{1}$ red and the remaining vertices blue) and $\chi(H)=\chi_{D}(H)=3$ (using three different colors for $w_{1}, w_{2}$, and $w_{3}$, and coloring $v_{1}$ with $w_{1}$ 's color).

Since a proper distinguishing coloring of a graph is both a proper coloring and a distinguishing labeling, we get the lower bounds $\chi_{D}(G) \geq \chi(G)$ and $\chi_{D}(G) \geq D(G)$. We also have the following simple upper bound.

Proposition 1.1 For any graph $G$ we have $\chi_{D}(G) \leq \chi(G) D(G)$.

Proof. Let $G$ be a graph and fix a proper coloring of $G$ using $\chi(G)$ colors $f: V(G) \rightarrow$ $\{1,2,3, \ldots, \chi(G)\}$ and a distinguishing labeling of $G$ using $D(G)$ labels $g: V(G) \rightarrow$ $\{1,2,3, \ldots, D(G)\}$. Then the labeling that assigns the ordered pair $(f(v), g(v))$ to vertex $v$ is a proper distinguishing coloring and uses $\chi(G) D(G)$ labels.

The bound in Proposition 1.1 is sharp. For example, the graph $C_{6}$ has $\chi\left(C_{6}\right)=2$, $D\left(C_{6}\right)=2$ and $\chi_{D}\left(C_{6}\right)=4$. Also, any graph $G$ with either $\chi(G)=1$ or $D(G)=1$ will have $\chi(G) D(G)=\chi_{D}(G)$.

In [4], the first and third authors define the distinguishing chromatic number $\chi_{D}(G)$ of a graph $G$ and prove results about $\chi_{D}(G)$ based on the underlying graph $G$. In particular, they find $\chi_{D}(G)$ for various families of graphs and prove analogues of Brooks' Theorem for both the distinguishing number and the chromatic distinguishing number. In this paper we approach the subject from the perspective of group theory and prove results about $\chi_{D}(G)$ based on the automorphism group of $G$. Since the publication of [4], the topic of 
the distinguishing chromatic number has been studied by other authors, for example, see $[3,15,16]$.

We end this section with a few definitions. If $G$ and $H$ are isomorphic graphs we write $G \approx H$. An $r$-coloring of a graph is a coloring of the vertices using $r$ colors. A $p$-group is a group whose order is a power of $p$, where $p$ is prime. The automorphism group of the colored graph $G$ (or just of the coloring of $G$ ) is the subgroup of $\operatorname{Aut}(G)$ that preserves vertex colors. If $\sigma$ is an automorphism of graph $G=(V, E)$ and $X \subseteq V$ then we define $\sigma(X)=\{\sigma(x): x \in X\}$.

\section{Graphs with $\chi_{D}(G) \leq 2$}

The main result of this section, Theorem 2.6, characterizes the automorphism groups of graphs with $\chi_{D}=2$. We begin with two elementary remarks.

Remark 2.1 $\chi_{D}(G)=1$ if and only if $G=K_{1}$. In this case, $\operatorname{Aut}(G)=\{i d\}$.

Remark 2.2 A connected bipartite graph can be properly 2-colored in exactly 2 ways: the coloring is forced once any one vertex's color is fixed.

Lemma 2.3 Suppose $G$ is a connected graph with $\chi_{D}(G)=2$. Then there is a unique proper red/blue coloring of the vertices of $G$ (up to reversing all vertex colors) and it is distinguishing. Furthermore, any nontrivial automorphism of $G$ must interchange red with blue vertices.

Proof. We know that $G$ is bipartite since $\chi(G) \leq \chi_{D}(G)=2$. By Remark 2.2, there is a unique proper 2-coloring of the vertices of $G$ (up to reversing all vertex colors). Therefore, this coloring must be distinguishing.

To justify the final sentence of the lemma, let $\sigma$ be a nontrivial automorphism of $G$. Since our coloring is distinguishing, without loss of generality, $\sigma$ maps a red vertex $x$ to a blue vertex $y$. However, automorphisms preserve distance, so once one red vertex is mapped to a blue one, all red vertices must be mapped to blue ones and vice versa. Thus $\sigma$ interchanges red and blue vertices.

Theorem 2.4 If $G$ is connected and $\chi_{D}(G)=2$ then the automorphism group of $G$ is either the identity or $\mathbf{Z}_{2}$.

Proof. Suppose $\operatorname{Aut}(G)$ is not the identity. Let $\sigma$ and $\tau$ be non-trivial automorphisms of $G$, not necessarily distinct. By Lemma 2.3, $\sigma$ and $\tau$ both interchange red and blue vertices. But then $\sigma \tau$ takes red vertices to red vertices, so must be the identity. Hence $\tau=\sigma^{-1}$, so $\operatorname{Aut}(G)$ has at most 3 elements: $i d, \sigma, \sigma^{-1}$. When $\tau=\sigma$, we see that $\sigma=\sigma^{-1}$. Hence $\operatorname{Aut}(G)$ is $\mathbf{Z}_{2}$, as required.

Note that if we only assume that $\chi(G)=2$, then the automorphism group of $G$ can be any group, see [12]. 
Lemma 2.5 If $G$ is a graph with $\chi_{D}(G)=2$ then the following are true:

1. There can not be three isomorphic components of $G$.

2. In any proper 2-distinguishing coloring of $G$, pairs of isomorphic components must be colored oppositely and the automorphism group of each of these components is trivial.

Proof. If $G$ has 3 isomorphic components, then by Remark 2.2, two of these components must be colored the same. Thus, there is a non-trivial automorphism of $G$ that interchanges these two components and preserves colors, a contradiction. This proves (1).

Next we prove (2). Fix a proper 2-distinguishing coloring of $G$. Let $J_{1}$ and $J_{2}$ be two isomorphic components of $G$. If $J_{1}$ and $J_{2}$ are colored identically then there is a non-trivial automorphism of $G$ that interchanges $J_{1}$ and $J_{2}$ and preserves the colors, a contradiction. Otherwise, by Remark 2.2, $J_{1}$ and $J_{2}$ are colored oppositely, as desired.

Finally, we prove the second part of (2). For a contradiction, suppose there is a nontrivial automorphism $\sigma_{1}$ of $J_{1}$ and let $\sigma_{2}$ be the corresponding automorphism of $J_{2}$. Let $\sigma$ be the automorphism of $G$ which acts on $J_{1}$ by $\sigma_{1}$ and on $J_{2}$ by $\sigma_{2}$ and fixes the remaining vertices of $G$. By Lemma 2.3, $\sigma$ interchanges red and blue vertices of $J_{1}$ and of $J_{2}$. Now let $\tau$ be the automorphism that interchanges $J_{1}$ and $J_{2}$ and fixes the rest of $G$. Hence $\tau$ interchanges red and blue vertices in $J_{1}$ and $J_{2}$. Then $\sigma \circ \tau$ is an automorphism of $G$ that preserves colors. However, $\sigma \circ \tau \neq i d$ because it interchanges vertices of $J_{1}$ with vertices of $J_{2}$. This is a contradiction.

Thus graphs with $\chi_{D}=2$ consist of unique components and pairs of isomorphic components. We can now extend Theorem 2.4 to the case where $G$ is not necessarily connected.

Theorem 2.6 If $\chi_{D}(G)=2$, then the automorphism group of $G$ is $\mathbf{Z}_{2} \times \mathbf{Z}_{2} \times \cdots \times \mathbf{Z}_{2}=\mathbf{Z}_{2}^{k}$ where $k$ is the number of pairs of isomorphic components plus the number of unique components in $G$ that have a non-trivial automorphism.

Proof. By Lemma 2.5, $G$ consists of components that are either unique or have one isomorphic duplicate. Let $A$ be the set of components that occur in pairs. By Theorem 2.4 the unique components either have a unique non-trivial automorphism (an involution), or have only the identity automorphism. Let $B$ be the set of components of $G$ that are unique and have a unique non-trivial involution and let $C$ be the set of components of $G$ that are unique but have only the identity automorphism. Let $k=|A|+|B|$. For each element of $A$, there is an involution of $G$, namely, the one which interchanges the two duplicates. Similarly, for each element of $B$, there is an involution of $G$, namely, its unique involution. Furthermore, these involutions act independently, and generate the automorphism group of $G$. Thus, $\operatorname{Aut}(G)=\mathbf{Z}_{2} \times \mathbf{Z}_{2} \times \cdots \times \mathbf{Z}_{2}=\mathbf{Z}_{2}^{k}$.

The next corollary follows directly from Theorem 2.6 and Remark 2.1.

Corollary 2.7 If $G$ is a graph and there exists an odd prime $p$ for which $|\operatorname{Aut}(G)|$ is divisible by $p$ then $\chi_{D}(G) \geq 3$. 


\section{Larger values of $\chi_{D}(G)$}

We have seen above that automorphism groups of graphs $G$ with $\chi_{D}(G)=2$ must be elementary abelian 2-groups. It is then natural to ask whether there are any restrictions on automorphism groups of graphs $G$ with $\chi_{D}(G)=r$. The answer given in the following theorem is no. Given any finite group $\Gamma$ and any integer $r \geq 3$, the same construction used in the proof of Theorem 3.1 yields a graph $G$ with $\operatorname{Aut}(G)=\Gamma$ and $\chi(G)=r$. However, Albertson and Collins [1] show that each graph $G$ with an abelian automorphism group has $D(G) \leq 2$. So Theorem 3.1 still holds if $\chi_{D}$ is changed to $\chi$ (and even if $r=2$ ), but does not hold if $\chi_{D}$ is changed to $D$.

Theorem 3.1 For any finite group $\Gamma$ and any integer $r \geq 3$, there exists a graph $G$ with $\operatorname{Aut}(G)=\Gamma$ and $\chi_{D}(G)=r$.

Proof. Given $\Gamma=\left\{\sigma_{0}=i d, \sigma_{1}, \sigma_{2}, \ldots, \sigma_{n}\right\}$ we construct a graph $G$ as follows: each nonidentity element $\sigma_{i} \in \Gamma$ is assigned a gadget. The gadget assigned to $\sigma_{k}$ consists of a path $P_{4}, v_{1}, v_{2}, v_{3}, v_{4}$ with a single vertex $x$ joined to $v_{3}$ and a path $v_{2}, y_{1}, y_{2}, \ldots, y_{k+2}$. Start with a graph $H$ with $V(H)=\Gamma$ and place a directed edge from $\sigma_{i}$ to $\sigma_{j}$ for all $i \neq j$. Replace the directed edge $\left(\sigma_{i}, \sigma_{j}\right)$ with the gadget assigned to $\sigma_{k}$ where $\sigma_{j}=\sigma_{i} \sigma_{k}$. In order to ensure that $\chi(G) \geq r$ (and thus $\chi_{D}(G) \geq r$ ) we add an extra $r-2$ vertices, $z_{1}, z_{2}, \ldots z_{r-2}$ to the $\sigma_{1}$-gadget to form an $r$-clique with $y_{1}$ and $y_{2}$ and add a path of length $i$ to each $z_{i}$ to eliminate automorphisms that swap $z$ 's. For an example with $r=4$ and $\Gamma=\mathbf{Z}_{3}=\left\{\sigma_{0}=i d, \sigma_{1}, \sigma_{2}\right\}$, see Figure 1 .

It has been shown [6] that $\operatorname{Aut}(G)=\Gamma$ and furthermore the action of $\sigma_{k} \neq i d$ on $G$ takes a $\sigma_{k}$ gadget to a different $\sigma_{k}$ gadget. It remains to show that $\chi_{D}(G) \leq r$. Color the vertices of $H$ red. For each $k>1$, color the $\sigma_{k}$ gadget from $i d$ to $\sigma_{k}$ as folows. Color vertex $v_{2}$ blue, and then finish the coloring of the bipartite gadget with blue and green. Color all the other $\sigma_{k}$ gadgets oppositely, with the base of the long chain colored green. Color the vertices of the $\sigma_{1}$ gadget similarly, use $r-2$ additional colors for the $z$ vertices and color the additional paths properly using blue and green. In Figure 1, the blue vertices are shown with a surrounding circle.

This is a proper coloring using $r$ colors. We must show it is distinguishing. Let $\tau=\sigma_{k}$ be a non-trivial automorphism of $G$. Then $\tau$ maps the $\sigma_{k}$ gadget between $i d$ and $\sigma_{k}$ to the one between $\sigma_{k}$ and $\sigma_{k}^{2}$ and thus does not preserve colors. Therefore our coloring is distinguishing.

Given a graph $H$, the difference between $\chi_{D}(H)$ and $\chi(H)$ arises from the automorphism group of $H$. We will study how large this difference can be in the next section. We now show that, no matter how large this difference is, there exists a graph $G$ so that $H$ is an induced subgraph of $G$ and $\chi_{D}(H) \geq \chi_{D}(G)$. This means that there can be no meaningful result that bounds the distinguishing chromatic number of a subgraph in terms of the distinguishing chromatic number of the larger graph.

Proposition 3.2 For any connected graph $H$ with $\chi(H)=k \geq 2$, there exists a graph $G$ with $\chi_{D}(G)=k$ and $\operatorname{Aut}(G)=\{i d\}$ containing $H$ as an induced subgraph. In particular, $\chi_{D}(H) \geq \chi_{D}(G)$. 


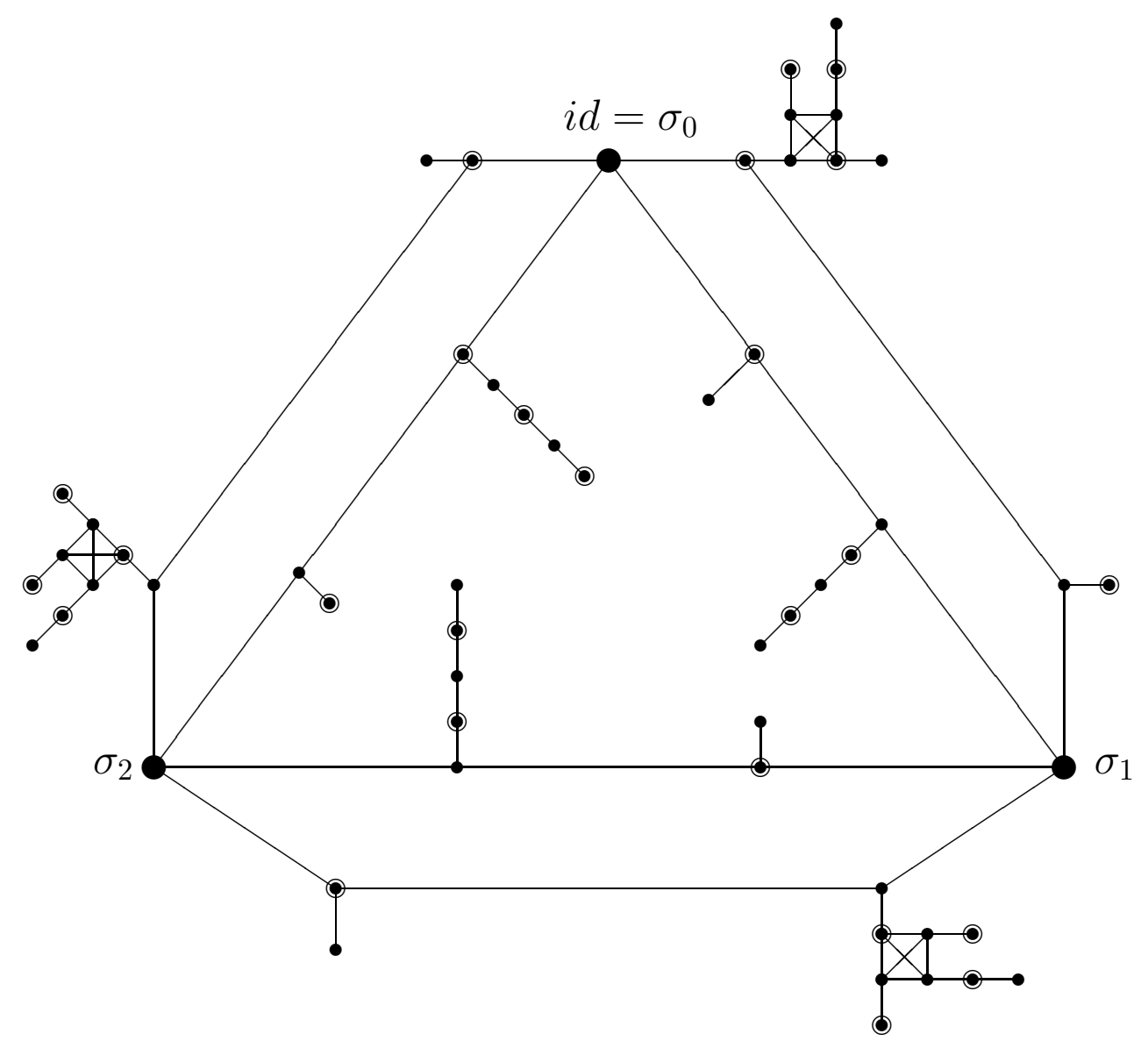

Figure 1: The graph constructed in Theorem 3.1 when $r=4$ and $\Gamma=\mathbf{Z}_{3}$.

Proof. Let $H$ be any connected graph with $\chi(H)=k \geq 2$. Order the vertices of $H$ by writing $V(H)=\left\{v_{1}, v_{2}, \ldots, v_{m}, w_{1}, w_{2}, \ldots, w_{n}\right\}$ where for each $i$, vertex $v_{i}$ is a leaf (i.e., $\operatorname{deg}\left(v_{i}\right)=1$ ) and $\operatorname{deg}\left(w_{i}\right) \geq 2$. For each $i$, add paths of length $2 i-1$ and $2 i$ to $v_{i}$ and a path of length $2 m+i$ to $w_{i}$. Let $G$ be the resulting graph (see Figure 2 for an example). By construction, $H$ is induced in $G$ and $\chi(G)=k$.

It remains to show that $\chi_{D}(G)=k$. Let $\sigma$ be any automorphism of $G$. In the graph $G$, vertices of $H$ have degree at least 3 while vertices in $V(G)-V(H)$ have degree at most 2, thus $\sigma$ maps vertices of $H$ to vertices of $H$ and leaves to leaves. By construction, each leaf of $G$ has a distinct distance to the closest vertex of degree 3 or more, so each leaf is mapped to itself, and this forces $\sigma$ to be the identity. Thus $\operatorname{Aut}(G)=\{i d\}$ and $\chi_{D}(G)=\chi(G)=k$ as desired.

The last sentence of the theorem follows since $\chi_{D}(H) \geq \chi(H)=k$.

One might wonder if it is possible to generalize Proposition 3.2 by also specifying the automorphism group of the graph $G$ of which $H$ is to be an induced subgraph. We carry 


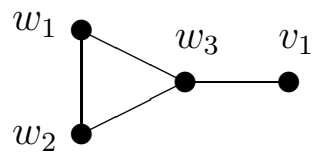

$H$

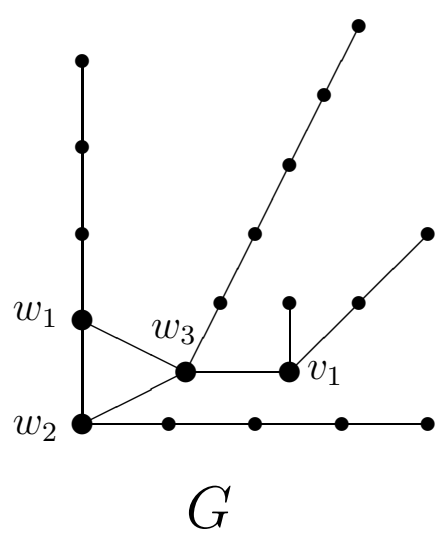

Figure 2: The graph $G$ constructed from $H$ in Proposition 3.2.

this out for $\operatorname{Aut}(G)=\mathbf{Z}_{2}$. We suspect it can be done for any value of $\operatorname{Aut}(G)$ as long as we assume $\chi(H)>2$.

Proposition 3.3 For any connected graph $H$ with $\chi(H)=k \geq 2$, there exists a graph $G^{\prime}$ with $\chi_{D}\left(G^{\prime}\right)=k$, Aut $\left(G^{\prime}\right)=\mathbf{Z}_{2}$ and $H$ is an induced subgraph of $G^{\prime}$.

Proof. Let $H$ be any connected graph with $\chi(H)=k \geq 2$ and let $G$ be the graph constructed from $H$ in the proof of Proposition 3.2. Form $G^{\prime}$ by taking two copies of $G$ and joining one pair of corresponding vertices $x$ and $x^{\prime}$. By construction, $H$ is induced in $G^{\prime}$ and $\chi\left(G^{\prime}\right)=k$. We showed that $\operatorname{Aut}(G)=\{i d\}$ in the proof of Proposition 3.2, thus the only nontrivial automorphism $\sigma$ of $G^{\prime}$ swaps the two copies of $G$ and switches $x$ and $x^{\prime}$. There is an edge between vertices $x$ and $x^{\prime}$ in $G^{\prime}$, so these vertices have different colors and therefore $\sigma$ does not preserve colors. Hence the only automorphism of $G^{\prime}$ that preserves colors is the identity and thus $\chi_{D}\left(G^{\prime}\right)=\chi\left(G^{\prime}\right)=k$ as desired.

\section{Bounds on $\chi_{D}(G)-\chi(G)$}

Given a finite group $\Gamma$, one can look at the maximum value $A(\Gamma)$ of the difference $\chi_{D}(G)-$ $\chi(G)$ for graphs $G$ with $\operatorname{Aut}(G)=\Gamma$. In this section, we show that $A(\Gamma)=1$ for cyclic groups of prime power order, and give upper bounds for $A(\Gamma)$ for general finite groups $\Gamma$ and also for finite abelian groups $\Gamma$. In the next section, we will prove that these bounds are tight.

Theorem 4.1 If $\operatorname{Aut}(G)=\mathbf{Z}_{2}$, then $\chi_{D}(G) \leq \chi(G)+1$.

Proof. Color $G$ using $\chi(G)$ colors. Let $\sigma$ be any non-trivial automorphism of $G$, which is an involution. If $\sigma$ does not preserve colors, then our coloring is distinguishing and 
$\chi_{D}(G)=\chi(G)$. If $\sigma$ does preserve colors, then recolor one of the vertices that is not fixed by $\sigma$ to be a new color. This gives a $(\chi(G)+1)$-distinguishing coloring of $G$.

By being slightly more careful with this proof, we get the following theorem.

Theorem 4.2 If $\operatorname{Aut}(G)=\mathbf{Z}_{p^{m}}$ for some prime $p$ and integer $m>0$, then $\chi_{D}(G) \leq$ $\chi(G)+1$.

Proof. Color $G$ using $\chi(G)$ colors. Let $\sigma$ be a generator of $\operatorname{Aut}(G)$. Then $\tau=\sigma^{p^{m-1}}$ is a nontrivial automorphism of $G$, and $\tau$ generates the unique subgroup $\Sigma$ of $\operatorname{Aut}(G)$ of order $p$. Thus $\tau$ is a power of any nontrivial element of $\Sigma$. Let $\omega$ be any nontrivial automorphism of $\operatorname{Aut}(G)$ with order $p^{t}$ for $t \geq 2$. Then $\omega^{p^{t-1}} \in \Sigma$, since $\omega^{p^{t-1}}$ has order $p$. Hence $\tau$ is a power of $\omega$. However, $\tau \neq i d$, so there exists a vertex $v$ that $\tau$ moves. Thus each $\omega$ must also move $v$. Now recolor $v$ with a new color. This gives a $(\chi(G)+1)$-distinguishing coloring of $G$.

To get a bound on $A(\Gamma)$ for more general finite groups $\Gamma$, we would like to iterate this method. Some problems arise, however, so the best we can do for a general group $\Gamma$ is the following.

Theorem 4.3 Suppose $\Gamma$ is a group of order $n$, and $G$ is a graph with $\operatorname{Aut}(G)=\Gamma$. Let $n=p_{1}^{i_{1}} p_{2}^{i_{2}} \cdots p_{k}^{i_{k}}$ where $p_{1}, \ldots, p_{k}$ are distinct primes. Then

$$
\chi_{D}(G) \leq \chi(G)+i_{1}+i_{2}+\cdots+i_{k} .
$$

Proof. Begin with a proper coloring of $G$ with $\chi(G)$ colors. We will recolor one vertex at a time with a completely new color to reduce the automorphism group of the colored graph $G$. For a number $m$, let us denote by $\mu(m)$ the sum of the exponents in the prime decomposition of $m$, so in particular, $\mu(n)=i_{1}+i_{2}+\cdots+i_{k}$. At the $j$ th step, we will have a proper coloring with $\chi(G)+j$ colors whose automorphism group $\Gamma_{j}$ has $\mu\left(\left|\Gamma_{j}\right|\right) \leq \mu(n)-j$. By the last step, then, we will have a coloring with $\chi(G)+\mu(n)$ colors with trivial automorphism group. The base case of the induction is $j=0$, which is clear. For the induction step, choose any element $\sigma$ of $\Gamma_{j}$, and a vertex $x$ that is not fixed by $\sigma$. Give $x$ a completely new color. This gives a proper coloring of $G$ with $\chi(G)+j+1$ colors. Any automorphism that preserves colors must fix vertex $x$, thus the automorphism group $\Gamma_{j+1}$ must be a subgroup of the stabilizer group of $x$. This subgroup cannot be all of $\Gamma_{j}$, since $\sigma$ moves $x$. Hence $\Gamma_{j+1}$ is a proper subgroup of $\Gamma_{j}$, so its order is a proper divisor of $\left|\Gamma_{j}\right|$. Thus $\mu\left(\left|\Gamma_{j+1}\right|\right)<\mu\left(\left|\Gamma_{j}\right|\right)$, completing the induction step.

Theorem 5.6 shows that the bound provided in Theorem 4.3 is tight in cases where $1=i_{1}=i_{2}=\cdots i_{k}$, that is, when $n$ is the product of distinct primes. When $k=1$, $n=p^{r}$ and $\operatorname{Aut}(G)=\left(\mathbf{Z}_{p}\right)^{r}$, then Theorem 4.3 gives the bound $\chi_{D}(G) \leq \chi(G)+r$, but Theorem 4.2 gives the improved bound $\chi_{D}(G) \leq \chi(G)+1$. If we take advantage of the structure of abelian groups $G$ as products of prime-power cyclic factors $\left(\mathbf{Z}_{p}\right)^{r}$, we obtain in Theorem 4.4 a bound on $\chi_{D}(G)-\chi(G)$ in terms of the number of prime-power cyclic factors. For example, if $n=180=2^{2} 3^{2} 5^{1}$ and $|\operatorname{Aut}(G)|=n$ then Theorem 4.3 gives the bound $\chi_{D}(G) \leq \chi(G)+2+2+1$. However, for the same $n$, if $\operatorname{Aut}(G)=\mathbf{Z}_{\left(2^{2}\right)} \times \mathbf{Z}_{\left(3^{2}\right)} \times \mathbf{Z}_{5}$ 
then Theorem 4.4 gives the bound $\chi_{D}(G) \leq \chi(G)+3$; if $\operatorname{Aut}(G)=\mathbf{Z}_{2} \times \mathbf{Z}_{2} \times \mathbf{Z}_{\left(3^{2}\right)} \times \mathbf{Z}_{5}$ then Theorem 4.4 gives the bound $\chi_{D}(G) \leq \chi(G)+4$; and if $\operatorname{Aut}(G)=\mathbf{Z}_{2} \times \mathbf{Z}_{2} \times \mathbf{Z}_{3} \times \mathbf{Z}_{3} \times \mathbf{Z}_{5}$ then both Theorems 4.3 and 4.4 give the bound $\chi_{D}(G) \leq \chi(G)+5$.

Theorem 4.4 Suppose $\Gamma$ is an abelian group and $G$ is a graph with $\operatorname{Aut}(G)=\Gamma$, so that

$$
\Gamma=\operatorname{Aut}(G)=\mathbf{Z}_{p_{1}^{n_{1}}} \times \cdots \times \mathbf{Z}_{p_{k}^{n_{k}}}
$$

for some $k$ where $p_{1}, \ldots p_{k}$ are primes, not necessarily distinct. Then $\chi_{D}(G) \leq \chi(G)+k$, and this bound is tight.

The proof of Theorem 4.4 relies on two technical results, Proposition 6.1 and Theorem 6.2 which we present in Section 6 .

Proof. We will prove the tightness of the bound in Theorem 5.6. Given $G$, we will prove by induction on $r$ that there is a coloring of $G$ with $\chi(G)+r$ colors such that the automorphism group $\Gamma_{r}$ of the coloring has at most $k-r$ prime-power cyclic factors. The base case is $r=0$, where it is obvious. For the induction step, write

$$
\Gamma_{r} \cong C_{1} \times \cdots \times C_{k-r}
$$

where each $C_{i}$ is a prime-power cyclic factor, and $C_{1}=\mathbf{Z}_{p^{s}}$ has the maximal order of all the $C_{i}$. Let $\sigma$ denote a generator of $C_{1}$. There must be a vertex $x$ that $\sigma^{p^{s-1}}$ does not fix. If $\Gamma_{x}$ denotes the stabilizer of $x$, this means that $\Gamma_{x} \cap C_{1}=\{i d\}$, since $\sigma^{p^{s-1}}$ is in every nontrivial subgroup of $C_{1}$. By Theorem 6.2, we can write $\Gamma_{r}=C_{1} \times B$ where $\Gamma_{x} \subseteq B$. Now color $x$ with a new color. Then the automorphism group $\Gamma_{r+1}$ of the new coloring must be a subgroup of $\Gamma_{x} \subseteq B \cong C_{2} \times \cdots \times C_{k-r}$. Therefore $\Gamma_{r+1}$ has at most $k-r-1$ prime-power cyclic factors, completing the induction.

\section{$5 \quad$ Tightness of bounds on $\chi_{D}(G)-\chi(G)$}

The main result in this section is a set of examples constructed in Theorem 5.6. Given any finite abelian group $\Gamma$, written as a product of $k$ prime-power cyclic groups, we construct a graph $H$ whose automorphism group is $\Gamma$ and for which $\chi_{D}(H)=\chi(H)+k$. These examples show the tightness of the bounds in Theorems 4.3 and 4.4. We begin by constructing the graphs $G_{n, i}$ and later will form $H$ by taking a join of such graphs.

The following example shows the bound in Theorem 4.2 is tight for $n=p^{m}$.

Example 5.1 Given positive integers $n, i$, we first construct the graph $G_{n, i}$ and show $\chi\left(G_{n, i}\right)=2, \chi_{D}\left(G_{n, i}\right)=3$, and $\operatorname{Aut}\left(G_{n, i}\right)=\mathbf{Z}_{n}$. To form the graph $G_{n, i}$, start with the even cycle $C_{2 n}$ with vertex set $V=\left\{x_{1}, x_{2}, \ldots, x_{2 n}\right\}$ and edges $x_{1} \sim x_{2} \sim \cdots x_{2 n} \sim x_{1}$. Replace every other edge of this cycle with a gadget as follows. For each $j: 1 \leq j \leq n$ replace the edge $x_{2 j-1}, x_{2 j}$ with the path $x_{2 j-1} \sim y_{2 j} \sim z_{2 j} \sim x_{2 j}$ where $y_{2 j}$ and $z_{2 j}$ are new vertices, add $i+1$ new vertices $u_{2 j, 1}, u_{2 j, 2}, \ldots, u_{2 j, i+1}$ to form the path $y_{2 j} \sim u_{2 j, 1} \sim$ 

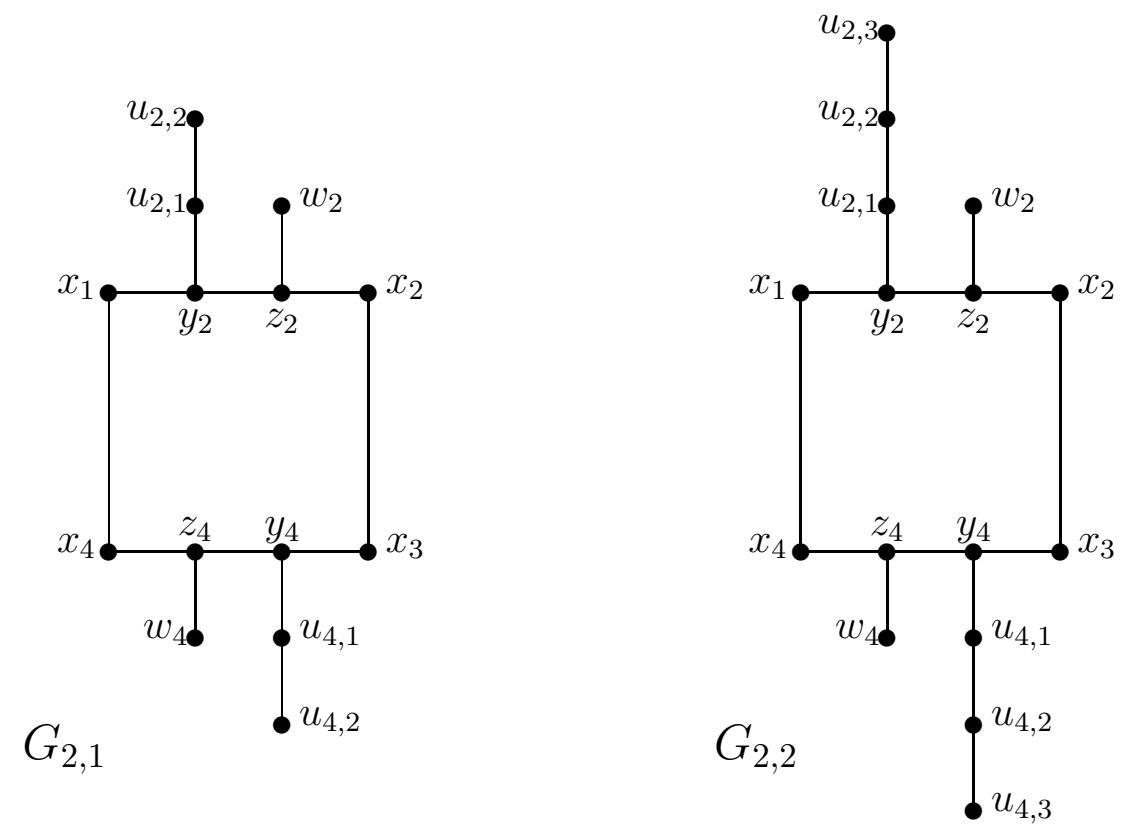

Figure 3: The graphs $G_{2,1}$ and $G_{2,2}$ from Example 5.1.

$u_{2 j, 2}, \cdots \sim u_{2 j, i+1}$ and one additional vertex $w_{2 j}$ with $z_{2 j} \sim w_{2 j}$. The graphs $G_{2,1}$ and $G_{2,2}$ are shown in Figure 3.

By construction, the graph $G_{n, i}$ contains only one cycle and that cycle is even; thus $G_{n, i}$ is bipartite and $\chi\left(G_{n, i}\right)=2$. The only automorphisms of $G_{n, i}$ are rotations that map $x_{1}$ to $x_{2 j-1}$ for some $j: 1 \leq j \leq n$, thus $\operatorname{Aut}\left(G_{n, i}\right)=\mathbf{Z}_{n}$. Since these rotations preserve any 2-coloring of $G_{n, i}$, we know $\chi_{D}\left(G_{n, i}\right)>2$. Using a new color for vertex $x_{1}$ gives a distinguishing coloring of $G_{n, i}$, and hence $\chi_{D}\left(G_{n, i}\right)=3$.

We now want to combine the graphs of the preceding example to construct a $G$ with $\operatorname{Aut}(G)=\mathbf{Z}_{p_{1}^{n_{1}}} \times \cdots \times \mathbf{Z}_{p_{k}^{n_{k}}}$ and $\chi_{D}(G)=\chi(G)+k$. The idea is to take the join of the graphs above.

Definition 5.2 The join of graphs $G_{1}, G_{2}, \ldots, G_{n}$, denoted by $G_{1} \vee G_{2} \vee \cdots \vee G_{n}$, has vertex set $V\left(G_{1}\right) \cup V\left(G_{2}\right) \cup \cdots \cup V\left(G_{n}\right)$ and edge set $E\left(G_{1}\right) \cup E\left(G_{2}\right) \cup \cdots \cup E\left(G_{n}\right) \cup\{x y \mid x \in$ $\left.V\left(G_{i}\right), y \in V\left(G_{j}\right), i \neq j\right\}$.

Our next Lemma shows that the automorphism group of the join of a particular set of graphs is the product of the automorphism groups of the individual graphs. Hemminger [7] addressed a more general version of this question using different notation. We include our proof for completeness.

Lemma 5.3 Suppose each of the graphs $G_{1}, G_{2}, \ldots, G_{n}$, is triangle free, and is not a complete bipartite graph, and also suppose $G_{i} \not \approx G_{j}$ whenever $i \neq j$. Then $\operatorname{Aut}\left(G_{1} \vee G_{2} \vee\right.$ $\left.\cdots \vee G_{n}\right)=\operatorname{Aut}\left(G_{1}\right) \times \operatorname{Aut}\left(G_{2}\right) \times \cdots \times \operatorname{Aut}\left(G_{n}\right)$. 
Proof. Let $G=G_{1} \vee G_{2} \vee \cdots \vee G_{n}$. If $\sigma_{i}$ is an automorphism of $G_{i}$ for $1 \leq i \leq n$ then define an automorphism $\sigma$ of $G$ by $\sigma(v)=\sigma_{i}(v)$ for $v \in V\left(G_{i}\right)$. This construction makes $\operatorname{Aut}\left(G_{1}\right) \times \operatorname{Aut}\left(G_{2}\right) \times \cdots \times \operatorname{Aut}\left(G_{n}\right) \subseteq \operatorname{Aut}(G)$.

Now let $\sigma$ be an automorphism of $G$. It remains to show that $\sigma$ induces an automorphism of $G_{i}$ for each $i$. First suppose there exist $u_{1} \in V\left(G_{i}\right), u_{2} \in V\left(G_{j}\right), u_{3} \in V\left(G_{\ell}\right)$ for distinct $i, j, \ell$ with $\sigma\left(u_{1}\right), \sigma\left(u_{2}\right), \sigma\left(u_{3}\right) \in V\left(G_{k}\right)$. Then $u_{1}, u_{2}, u_{3}$ form a triangle in $G$ hence $\sigma\left(u_{1}\right), \sigma\left(u_{2}\right), \sigma\left(u_{3}\right)$ form a triangle in $G_{k}$, a contradiction. Thus vertices sent to $G_{k}$ by $\sigma$ come from at most two distinct sets $G_{i}, G_{j}$.

We next show that if $\sigma$ sends vertices from $G_{i}$ and $G_{j}$ to $G_{k}$ for $i \neq j$ then those sets of vertices must be independent sets in $G_{i}$ and in $G_{j}$. Suppose there exist vertices $x, y \in V\left(G_{i}\right)$ and $z \in V\left(G_{j}\right)$ with $i \neq j$, and $x y \in E\left(G_{i}\right)$ and $\sigma(x), \sigma(y), \sigma(z) \in V\left(G_{k}\right)$. Then since $i \neq j$, vertex $z$ forms a triangle in $G$ with $x$ and $y$ which gives the triangle $\sigma(x), \sigma(y), \sigma(z) \in G_{k}$.

Thus for each $k$ there exists an $i$ and possibly a $j$ so that $V\left(G_{k}\right)$ can be partitioned as $V\left(G_{k}\right)=\sigma(X) \cup \sigma(Y)$ where $X \subseteq V\left(G_{i}\right)$ and $Y \subseteq V\left(G_{j}\right)$. We have shown that $X$ and $Y$ are independent sets in $G$. However, $x y \in E(G)$ for each $x \in X$ and each $y \in Y$ by the definition of $G$, so if $X$ and $Y$ are both non-empty then $G_{k}$ is a complete bipartite graph, a contradiction.

Hence for each destination $k$ there exists a unique origin $i$ so that $V\left(G_{k}\right)=\sigma(X)$ for some $X \subseteq V\left(G_{i}\right)$. Indeed, we must have $X=V\left(G_{i}\right)$ since there are exactly $n$ origins and $n$ destinations and $\sigma$ is an automorphism. Thus for each $k$ there exists an $i$ so that $\sigma\left(V\left(G_{i}\right)\right)=V\left(G_{k}\right)$. Since we are given $G_{i} \not \approx G_{k}$ for $i \neq k$ we must have $i=k$. This completes the proof that $\sigma \subseteq \operatorname{Aut}\left(G_{1}\right) \times \operatorname{Aut}\left(G_{2}\right) \times \cdots \times \operatorname{Aut}\left(G_{n}\right)$.

Lemma 5.4 For any graphs $G_{1}, G_{2}, \ldots, G_{n}$, we have $\chi_{D}\left(G_{1} \vee G_{2} \vee \cdots \vee G_{n}\right) \geq \chi_{D}\left(G_{1}\right)+$ $\chi_{D}\left(G_{2}\right)+\cdots+\chi_{D}\left(G_{n}\right)$.

Proof. Let $G=G_{1} \vee G_{2} \vee \cdots \vee G_{n}$. Any proper distinguishing coloring of $G$ induces a proper distinguishing coloring of each $G_{i}$. However, in $G$, each vertex of $G_{i}$ is adjacent to each vertex of $G_{j}$ for $i \neq j$, so the set of colors used for $G_{i}$ must be disjoint from the set used for $G_{j}$. Therefore, we need at least $\chi_{D}\left(G_{1}\right)+\chi_{D}\left(G_{2}\right)+\cdots+\chi_{D}\left(G_{n}\right)$ colors for $G$.

Corollary 5.5 If $G_{1}, G_{2}, \ldots, G_{n}$ are graphs with $\operatorname{Aut}\left(G_{1} \vee G_{2} \vee \cdots \vee G_{n}\right)=\operatorname{Aut}\left(G_{1}\right) \times$ $\operatorname{Aut}\left(G_{2}\right) \times \cdots \times \operatorname{Aut}\left(G_{n}\right)$, then $\chi_{D}\left(G_{1} \vee G_{2} \vee \cdots \vee G_{n}\right)=\chi_{D}\left(G_{1}\right)+\chi_{D}\left(G_{2}\right)+\cdots+\chi_{D}\left(G_{n}\right)$.

Proof. Let $G=G_{1} \vee G_{2} \vee \cdots \vee G_{n}$. By Lemma 5.4 we need only show $\chi_{D}(G) \leq$ $\chi_{D}\left(G_{1}\right)+\chi_{D}\left(G_{2}\right)+\cdots+\chi_{D}\left(G_{n}\right)$. Fix a distinguishing coloring of each $G_{i}$ so that the colors used in $G_{i}$ are disjoint from those used in $G_{j}$ whenever $i \neq j$. We claim that this is a distinguishing coloring of $G$. If not, there would be a non-trivial automorphism $\sigma$ of $G$ that preserves these colors. So $\sigma \in \operatorname{Aut}(G)=\operatorname{Aut}\left(G_{1} \vee G_{2} \vee \cdots \vee G_{n}\right)=$ $\operatorname{Aut}\left(G_{1}\right) \times \operatorname{Aut}\left(G_{2}\right) \times \cdots \times \operatorname{Aut}\left(G_{n}\right)$, and thus $\sigma$ preserves the colors of $G_{i}$ for each $i$. Since we chose distinguishing colorings of each $G_{i}$ we know that $\sigma$ is the identity automorphism on each $G_{i}$, hence $\sigma$ is the identity automorphism on $G$. This contradicts $\sigma$ being a non-trivial automorphism. 
We now have all the necessary ingredients to prove the tightness of the bound in Theorem 4.4. The same construction shows the bound in Theorem 4.3 is achieved by an abelian group.

Theorem 5.6 Given a finite abelian group

$$
\Gamma=\mathbf{Z}_{p_{1}^{n_{1}}} \times \cdots \times \mathbf{Z}_{p_{k}^{n_{k}}}
$$

there is a graph $H$ with $\operatorname{Aut}(H)=\Gamma$ and $\chi_{D}(H)=\chi(H)+k$.

Proof. Consider the graph

$$
H=G_{p_{1}^{n_{1}, 1}} \vee G_{p_{2}^{n_{2}, 2}} \vee \cdots \vee G_{p_{k}^{n_{k}, k}}
$$

Recall from Example 5.1 that $\chi_{D}\left(G_{p_{i} n_{i}, i}\right)=3$ for each $i$. Since $H$ is the join of $k$ graphs each having chromatic number 2 , we know $\chi(H)=2 k$. Note that for each $n$ and each $i$ the graph $G_{n, i}$ is triangle-free and is not a complete bipartite graph. Furthermore, $G_{n, i} \approx G_{m, j}$ only when $n=m$ and $i=j$. Therefore we may apply Lemma 5.3 to the graph $H$ to conclude

$$
\operatorname{Aut}(H)=\mathbf{Z}_{p_{1}^{n_{1}}} \times \cdots \times \mathbf{Z}_{p_{k}^{n_{k}}} .
$$

Now by Corollary 5.5, we have $\chi_{D}(H)=3 k$. Therefore $\chi_{D}(H)=\chi(H)+k$.

\section{Results about abelian groups}

In this section we present two technical results about abelian groups needed in the proof of Theorem 4.4. These results can be derived from theorems in [10] and [11].

Proposition 6.1 Suppose $A \leq B$ is an inclusion of finite abelian groups such that $p^{s} x=$ $i d$ for all $p$-torsion elements of $B$. Whenever $r \geq s$, any homomorphism $f: A \rightarrow \mathbf{Z}_{p^{r}}$ extends to a homomorphism $g: B \rightarrow \mathbf{Z}_{p^{r}}$.

Proof. We use the Prüfer group $\mathbf{Q} / \mathbf{Z}_{(p)}$. Recall that this group is an infinite $p$-group with a unique subgroup of order $p^{r}$ generated by $1 / p^{r}$. Let $j: \mathbf{Z}_{p^{r}} \rightarrow \mathbf{Q} / \mathbf{Z}_{(\mathbf{p})}$ denote the inclusion of this subgroup. Because $\mathbf{Q} / \mathbf{Z}_{(\mathbf{p})}$ is injective as an abelian group (see Section IV.3 of [8], there is an extension $g^{\prime}: B \rightarrow \mathbf{Q} / \mathbf{Z}_{(\mathbf{p})}$ of $j f$. Any element of $B$ that is not $p$-torsion is of course sent to $i d$ by this map. Any element of $B$ that is $p$-torsion is killed by $p^{s}$, so must land in the image of $j$ since $r \geq s$. Thus we can write $g^{\prime}=j g$ for some map $g: B \rightarrow \mathbf{Z}_{p^{r}}$, giving us the desired extension.

Theorem 6.2 Let $A$ be a finite abelian group acting on a set $X$. Let $C$ be a factor of $A$ that is isomorphic to $\mathbf{Z}_{p^{r}}$, where $p^{r}$ is the largest $p$-power order of an element of $A$. Suppose $x \in X$ is an element with $A_{x} \cap C=\{i d\}$, where $A_{x}$ is the stabilizer of $x$. Then there is a subgroup $B$ of $A$ such that $A_{x} \subseteq B$ and $A \cong C \times B$. 
Proof. By assumption, since $C$ is a factor of $A$, we have a splitting $A \cong C \times A / C$. This splitting is given by a retraction $\alpha: A \rightarrow C$ that fixes $C$, the projection $p: A \rightarrow A / C$, and a section $s: A / C \rightarrow A$ with $p s$ being the identity. So the splitting takes an element $a$ of $A$ to $(\alpha(a), p(a))$, and $\alpha(a)+s p(a)=a$. If the map $\alpha: A_{x} \rightarrow C$ were $i d$, then $A_{x}$ would lie in $s(A / C)$ and we would be done. In general, this will not be true, and we need to modify $s$ to make it be true. In general, if we replace $s$ by $s+t$ for some map $t: A / C \rightarrow C$, and replace $\alpha$ by $\alpha-t p$, then we will still get a splitting $A \cong C \times A / C$. Indeed, we still have $(\alpha-t p)(c)=c$ for $c \in C$, and $p(s+t)$ is still the identity, and

$$
(\alpha-t p)(a)+(s+t) p(a)=\alpha(a)+s p(a)=a,
$$

as required.

Now the map $p: A_{x} \rightarrow A / C$ has to be an injection, since any element in the kernel would have to be in both $A_{x}$ and $C$. By Proposition 6.1, there is a map $t: A / C \rightarrow C$ such that $t(p a)=\alpha(a)$ for all $a \in A_{x}$. As above, this gives us a new splitting $A \cong C \times A / C \cong$ $C \times(s+t)(A / C)$, but now if $a \in A_{x}$, we have

$$
a=(\alpha-t p)(a)+(s+t) p(a)=(s+t) p(a) .
$$

Therefore, $A_{x} \subseteq(s+t)(A / C)$ as required.

\section{Conclusion}

We conclude with an open question and acknowledgements. In Section 4 we defined $A(\Gamma)$ to be the maximum value of $\chi_{D}(G)-\chi(G)$ for graphs with $\operatorname{Aut}(G)=\Gamma$. There are many questions that one can ask about the invariant $A(\Gamma)$ for finite groups $\Gamma$. We have seen that $A(\Gamma)$ is bounded by some feature of $\Gamma$ : when $\Gamma$ is any group, $A(\Gamma)$ is bounded by the number of prime factors of $|\Gamma|$, and when $\Gamma$ is an abelian group, then $A(\Gamma)$ equals the number of prime power cyclic factors of $\Gamma$. If $\Gamma$ is a solvable group, perhaps $A(\Gamma)$ could be bounded by the shortest length of the composition series of a solvable group into prime power cyclic factors. This would fit with the bound that we have for abelian groups. The smallest group where the bounds might be different is $D_{4}$, which has order 8 , so by Theorem $4.3, A\left(D_{4}\right) \leq 3$. The shortest length of a composition series of $D_{4}$ into cyclic factors is 2 , since $D_{4}$ contains a cyclic group of size 4 .

An anonymous referee pointed out that the work of Seress [13] implies that if a graph $G$ is vertex transitive and has a solvable automorphism group then $D(G) \leq 5$ and $\chi_{D}(G)-$ $\chi(G) \leq 4$. Indeed, because $G$ is vertex transitive, the action of $\operatorname{Aut}(G)$ is primitive. Seress proves in this case that there is a set $S$ of size at most 4 such that any nontrivial automorphism of $G$ moves a vertex in $S$. To see that $D(G) \leq 5$ we color each vertex in $S$ differently and the remaining vertices get one new color. Similarly, to show $\chi_{D}(G) \leq$ $4+\chi(G)$, start with a proper coloring of $G$ and give each vertex of $S$ a new color.

The authors are grateful to both referees for their helpful comments and to Constance Leidy for suggesting Proposition 1.1 and its proof. 


\section{References}

[1] M. O. Albertson and K. L. Collins, Symmetry breaking in graphs, Electron. J. of Combin., 3 (1996), \#R18.

[2] C. T. Cheng and L. J. Cowen, On the local distinguishing numbers of cycles, Discrete Math. 196 (1999), no. 1-3, 97-108.

[3] J. O. Choi, S. G. Hartke and H. Kaul, The distinguishing chromatic number of cartesian products of graphs, preprint.

[4] K. L. Collins and A. N. Trenk, The distinguishing chromatic number, Electron. J. of Combin., 13 (2006), \#R16.

[5] J. Gross and J. Yellen, editors, Handbook of Graph Theory, CRC Press, Boca Raton, 2004.

[6] R. Frucht, Herstellung von Graphen mit vorgegebener abstrakter Gruppe, Compositio Math. 6 (1939), 239-250.

[7] R. Hemminger, The group of an $X$-join of graphs, J. Combinatorial Theory 5 (1968), $408-418$.

[8] T. W. Hungerford, Algebra, Graduate Texts in Mathematics, vol. 73, Springer-Verlag, New York, 1980, Reprint of the 1974 original.

[9] S. Klavžar, T. Wong and X. Zhu, Distinguishing labellings of group action on vector spaces and graphs, J. Algebra 303 (2006), no. 2, 626-641.

[10] R. S. Pierce, Centers of purity in abelian groups, Pacific J. Math. 13 (1963), 215-219.

[11] J. D. Reid, On subgroups of an Abelian group maximal disjoint from a given subgroup, Pacific J. Math. 13 (1963), 657-664.

[12] G. Sabidussi, Graphs with given group and given graph-theoretical properties, Canad. J. Math. 9 (1957), 515-525.

[13] Á. Seress, The minimal base size of primitive solvable perutation groups, J. London Math. Soc. (2) 53 (1996), 243-255.

[14] J. Tymoczko, Distinguishing numbers for graphs and groups, Electronic Journal of Combinatorics 11 (2004), \#R63.

[15] M. E. Watkins and X. Zhou, Distinguishability of locally finite trees, Electronic Journal of Combinatorics 14 (2007), \#R29.

[16] J. Weigand and M. S. Jacobson, Distinguishing and distinguishing chromatic numbers of generalized Petersen Graphs, AKCE J. Graphs. Combin., 5 no. 2, (2008), 199-211. 